\title{
Testing Equation Method Modification for Demanding Energy Measurements Verification
}

\author{
Kochneva Elena ${ }^{1}$, Pazderin Andrew ${ }^{1}$ and Sukalo Alexandar ${ }^{2}$ \\ ${ }^{1}$ Automated Electrical Systems Department, Ural Federal University named after the first President of Russia B.N. Yeltsin, \\ Yekaterinburg, Russia \\ ${ }^{2}$ Elektroperenos-Elektroprijenos BiH, Banja Luka, Bosnia i Hercegovina
}

\begin{abstract}
The paper is devoted to the mathematical approaches of the measurements received from Automatic Meter Reading Systems verification. Reliability of metering data can be improved by application of the new issue named Energy Flow Problem. The paper considers demanding energy measurements verification method based on verification expressions groups analysis. Bad data detection and estimates accuracy calculation is presented using the Automatic Meter Reading system data from the Russian power system fragment.
\end{abstract}

\section{Introduction}

Electrical Energy (EE) measurements received from Automatic Meter Reading (AMR) systems are used to provide financial settlements on EE markets and also in calculations of various technical and economic parameters for solving power system development and planning. The current AMR systems development trend is directed to the intelligent digital devices using, as well as increasing the number of AMR complexes. Despite the considerable technical improvement of AMR systems the level of $\mathrm{EE}$ losses (primary the commercial losses) remains at a significant level. Rapid bad data detection is a difficult task that allows to identify AMR complexes with errors that exceeds the maximum allowable limits and to locate the potential sources of commercial EE losses.

Currently there are significant number of approaches for metering data validation [1]-[8]. Metrological control is the most legitimate method of EE measurements reliability assessing. Metrological inspections are carried out once in several years and are based on approved methodologies and procedures. If there are distortion of metrological characteristic during the calibration interval metering data will contain error up to the end of recalibration interval. More often than once in several years the reliability evaluation can be carried out by calculation and analysis of actual and allowable EE imbalances. One of the main drawbacks of the balance technique is the inability to determine the concrete source of bad data. Mathematical methods of EE measurements reliability increasing are the most promising and inexpensive. Research has shown that the verification methods developed in the frameworks of State Estimation
(SE) theory [1]-[5] can successfully be used for EE measurements validation [9]-[11].

Energy Flow Problem (EFP) is the new issue concerning the electric condition parameters calculation. EFP allows to determine EE flows and technical losses on all elements of equivalent circuit using EE measurements and equivalent circuit parameters. EFP state equations system consists of the system of EE nodal balances, the system of EE balance in network branches and equations of technical losses calculation [9]. SE theory methods adopted to EE measurements in the frameworks of EFP allows to solve a variety of tasks such as determining the required amount and optimal location of AMR complexes for observability provision [12], determining the sources of EE commercial losses. This paper presents the method of Verifying Equations (VE) for demanding energy measurements verification

\section{Theoretical background}

Some EE measurements require more careful verification. These measurements include commercial ones, i.e. the measurements used for financial transactions, and important interconnections EE measurements. Additional approaches can be applied to such demanding measurements verification.

One of the promising approaches is testing equations (TE) technique. The core of the method and the process of TE system construction is discussed in details in [10]. $\mathrm{TE}$ are balance equations for any electrical network fragment that contain only actual EE measurements [8], [10]. TE imbalance occurs due to measurements errors presence. Any measurement error evaluation can be carried out using TE method by forming the group of 
linearly independent VE. Each measurement can be calculated through a linear combination of a number of other measurements in many ways using TE system. The number of such equations depends on the dimension of TE system. The previous papers demonstrate the number of TE is determined by metering system redundancy. The minimum number of measurements that provide observability of active (reactive) EE distribution is equal to the number of branches in network diagram [9]. If there is no duplicate measurements all the rest ones are redundant. The number of redundant measurements is equal to the number of TE in system. If there is no redundant measurements it is impossible to construct TE system and to provide mathematical measurements verification.

Construction of the VE is considered in five nodal network presented in Fig. 1. For clarity there is no measurement errors and no technical losses.

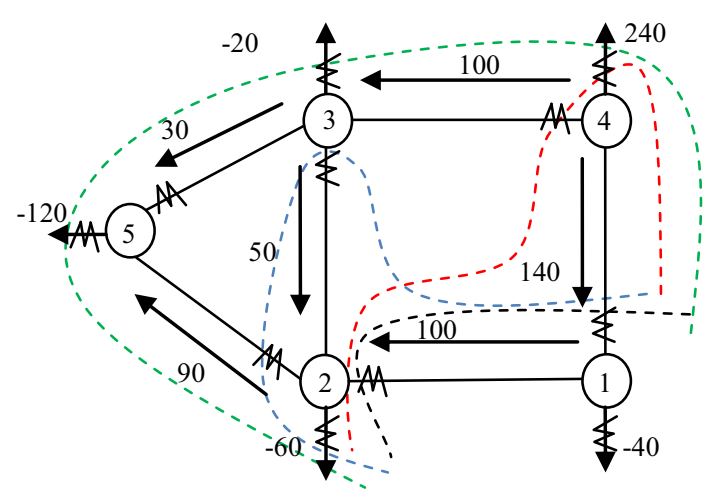

Figure 1. AMR complexes, measurements

There is 6 branches in the network under consideration. hence $6 \mathrm{EE}$ measurements provide observability. There is 11 measurements in the scheme so $11-6=5$ measurements are redundant and it is possible to draw up a system of five TE. Five VE can be received using TE system. Such VE are presented for nodal measurement $W_{l}$. Similarly to drawing up the system of TE, VE can be formed in two ways (algebraic and topological method). The algebraic method is more formalized. VE are formed by converting the TE system:

$$
\left\{\begin{array}{l}
-W_{1}=-W_{1-2}+W_{1-4}, \\
-W_{2}=W_{1-2}+W_{2-3}-W_{2-5} \\
-W_{3}=W_{3-4}-W_{2-3}-W_{3-5} \\
-W_{4}=-W_{1-4}-W_{3-4} \\
-W_{5}=W_{3-5}+W_{2-5} .
\end{array}\right.
$$

The first VE is equation number 1 from (1). Here the measured $\mathrm{EE}$ values are substituted to the right side:

$$
W^{(1)}{ }_{1}=-\left(W_{1-2}-W_{1-4}\right)=-(-100+140)=-40
$$

This VE is the basis for the others. EE measurements along the branches 1-2 and 1-4 can be expressed through other measurements and substituted to the first VE.

$$
W_{1}^{(2)}=-\left(W_{4}-W_{4-3}-W_{1-4}\right)=-(240-100-100)=-40,
$$

here the forth equation from (1) is used. Measurement of branch 1-4 was expressed through $W_{4}$ and $W_{3-4}$.

The third VE can be obtained by expressing $W_{1-2}$ from the second equation of (1):

$$
W_{1}^{(3)}=-\left(W_{1-4}+\left(W_{2}-W_{2-5}+W_{3-2}\right)=-(140+(-60-90+50))=-40 .\right.
$$

The forth VE is recieved likewise:

$$
\begin{aligned}
& W_{1}^{(4)}=-\left(W_{4}-W_{4-3}+\left(W_{2}-W_{2-5}+W_{3-2}\right)=\right. \\
& =-(240-100+(-60-90+50))=-40 .
\end{aligned}
$$

Last VE is obtained by summing all equations from (1):

$$
W_{1}^{(5)}=-\left(W_{2}+W_{3}+W_{4}+W_{5}\right)=-(-60-120-20+240)=-40 \text {. }
$$

There is no measurements errors in this hypothetical example, so all VE yield a result which coincides with the actual value $W_{1}=-40$.

$\mathrm{VE}$ can be constructed for any nodal or linear measurements. Topological technique of VE construction is more vivid. Topological interpretation of VE composed by algebraic technique is presented as dotted lines in Fig. 1 .

Thus TE system application for VE groups construction allows to calculate any measurement through linear combination of the other measurements. The difference in the VE calculations corresponds to reliability of the measurement selected for VE. This approach allow to improve the reliability of demanding EE measurements.

\section{Case study}

Fragment of EE system is presented in Figure 2.The network consists of four-nodal ring and three radial sections. Locations of AMR complexes are marked by X. The number of nodes (without null) is 10 , the number of branches is 10 . There are 13 measurements in the network. 13 redundant measurements allow to get $13 \mathrm{TE}$ and $13 \mathrm{VE}$.

Redundant measurements set allows to implement verification methods in particular TE technique. The source of metering data is AMR system installed in the 
affected substations. Calculations were performed for 1440 hour intervals.

EFP state equations system was performed for tje network presented in Fig. 2. Elimination of all

$$
\begin{aligned}
& W_{1}^{1}=W_{2}-\Delta W_{1-13} ; \\
& W_{1}^{2}=-\left(W_{4}+W_{5}+W_{12}+W_{13}+W_{14}+W_{15}+W_{16}+W_{3}+\right. \\
& \left.+W_{10}+W_{11}\right)-\Delta W_{1-13} ; \\
& W_{1}^{3}=-\left(W_{4}+W_{5}+W_{12}+W_{13}+W_{14}+W_{15}+W_{16}+W_{3}+\right. \\
& \left.+W_{10}+W_{34}-\Delta W_{1-6(I I)}\right)-\Delta W_{1-13} ; \\
& W_{1}^{13}=W_{6}+W_{7}+W_{8}+W_{9}+W_{39}+W_{40}+W_{19}+W_{20}+W_{21}+ \\
& +W_{22}+W_{11}+W_{25}+W_{24}+W_{29}+W_{28}+W_{27}+W_{31}+W_{30}+ \\
& +W_{32}+W_{37}+W_{38}+W_{36}+\Delta W_{1-111-112}+\Delta W_{1-121-122}+\Delta W_{72-7111}+ \\
& +\Delta W_{1-7}+\Delta W_{1-2(I)}+\Delta W_{1-2(I I)}+\Delta W_{2-212-2111}+\Delta W_{2-221-2221}+ \\
& \Delta W_{1-3}+\Delta W_{3-312-3111}+\Delta W_{1-4}+\Delta W_{4-422-4211}+\Delta W_{5-4}+ \\
& +\Delta W_{5-6}+\Delta W_{6-61}+\Delta W_{6-62}+\Delta W_{1-6(I)}+\Delta W_{1-6(I I)}+\Delta W_{1-13} .
\end{aligned}
$$

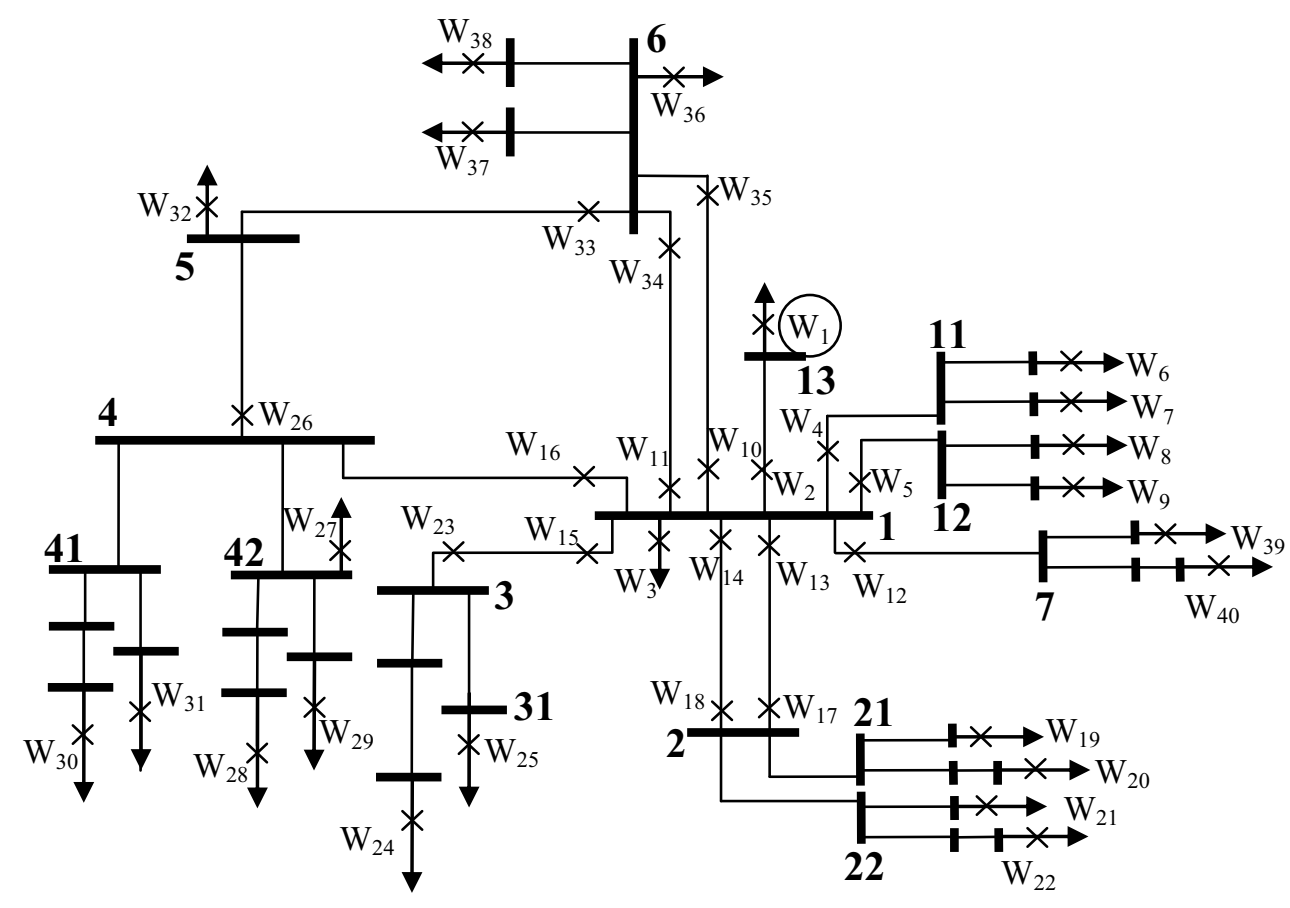

Figure 2. Case study. Network diagram, AMR complexes location

Imbalance error evaluation for each VE can be obtained on the basis of maximum permissible error of the constituent measurements:

$$
\delta_{W_{i}}=\sqrt{\sum_{j=1}^{n} a_{j}^{2} \cdot \delta_{W_{j}}^{2}}
$$

here $\delta_{W_{j}}$ is maximum permissible measuring error for the i-th AMR complex calculated according to fundamentals of metrology [13].

The value of maximum permissible measuring error can be seen as double value of standard deviation which is calculated on the basis of measuring instruments included in AMR complex accuracy classes.
Group (2) provides number of estimates calculation for the chosen demanding measurement on each measurement interval. So for the first hour actual measurement $W_{l}$ is $257600 \mathrm{kWh}$. Calculated estimates of $W_{1}$ according to each $\mathrm{VE}$ are presented in the second column of Table 1.

Table 1. Estimates and calculated errors of VE.

\begin{tabular}{|c|c|c|c|c|}
\hline VE & $\begin{array}{c}\text { VE } \\
\text { value, } \\
\mathbf{k W h}\end{array}$ & $\begin{array}{c}\text { Difference, } \\
\mathbf{k W h}\end{array}$ & $\begin{array}{c}\text { Difference, } \\
(\mathbf{r}), \mathbf{\%}\end{array}$ & $\begin{array}{c}\mathbf{V E} \\
\text { error, \% }\end{array}$ \\
\hline$W_{1}^{\text {calc(1) }}$ & 253894 & 3706 & 1,438 & 1,15 \\
\hline$W_{1}^{\text {calc(2) }}$ & 256446 & 1154 & 0,447 & 2,15 \\
\hline$W_{1}^{\text {calc(1) }}$ & 256969 & 5516 & 0,244 & 2,15 \\
\hline
\end{tabular}




\begin{tabular}{|c|c|c|c|c|}
\hline$\ldots \ldots \ldots$ & $\ldots \ldots \ldots$ & $\ldots \ldots \ldots$ & $\ldots \ldots \ldots$ & \\
\hline$W_{1}^{\text {calc(13) }}$ & 280893 & -23294 & $-9,042$ & 4,09 \\
\hline$W_{1}^{\text {final_calc }}$ & $\mathbf{2 5 4 6 2 3}$ & $\mathbf{2 9 7 7}$ & $\mathbf{1 , 1 6}$ & $\mathbf{0 , 5 9}$ \\
\hline
\end{tabular}

Third and fourth columns contain differences between actual measurement and estimates expressed in percentage and absolute value. Fifth column of Table 1 shows the evaluations of maximum permissible errors of VE.

Difference between actual measurement value and its calculated analogue allows to judge about this measurement reliability. The smaller the difference between actual and calculated measurement value the greater the confidence in the measurement. Analysis of Table 1 shows "steps" in VE errors. The more AMR complexes are involved into calculations the higher is the error of the separate VE. The first step is using the nodal EE balance. The second step (or belt) is formed by VE where each linear measurement from the first step VE is expresses through the measurement of the other branch end and technical losses value. Most often, the relative VE error of each "step" is about the same.

If the maximum permissible measuring errors of AMR complexes are the same, the error of each calculated analogues is exceed the error of the measurement. However the presence of a calculated analogues number obtained on the basis of VE allows to compute the final calculated estimate of the measurement by averaging all calculated analogues and taking the variances into account:

$$
W_{k}^{\text {final_calc }}=\frac{\sum_{i=1}^{N} \frac{1}{D_{i}} W_{k}^{\text {calc }(i)}}{\sum_{i=1}^{N} \frac{1}{D_{i}}}
$$

here $D_{i}$ is dispersion of $i$-th VE; $W_{k}^{\text {final-calc is final }}$ calculated estimate of the required measurement; $W_{k}^{\text {calc }(i)}$ is the value of $i$-th VE.

The final calculated value of $W_{l}$ obtained by averaging on the basis of (4) is 254623. The relative value of final calculated estimate error of $W_{1}$ is $0,59 \%$ which is almost two times less than the maximum permissible error of AMR complexes 1,1\%. The maximum permissible error of AMR complex is determined on the basis of accuracy classes of AMR complex components [13]:

$\delta_{W}= \pm 1,1 \sqrt{\delta_{U}^{2}+\delta_{I}^{2}+\delta_{C}^{2}+\delta_{\mathrm{L}}^{2}}= \pm 1,1 \sqrt{0,5^{2}+0,5^{2}+0,5^{2}+0,5^{2}}=1,1$, here $\delta_{I}, \delta_{U}, \delta_{C}$ are accuracy ratings of a current transformer, voltage transformer and an energy meter correspondingly; $\delta_{L}$ is a voltage drop in a cable line.

The presence of numerous intervals for calculation allows collecting statistical information about estimates error for each AMR complex. Current AMR systems have sufficient depth of archives to provide the required number of measurements. Subsequent processing of such statistical series can rate the characteristics of the concrete AMR complexes. AMR complex is considered in good condition if the average value of errors series does not exceed the maximum permissible error for this AMR complex.

Fig. 3 shows the graphical processing of calculation errors for two AMR complexes $3 \mathrm{a}$ and $3 \mathrm{~b}$. The shaded area corresponds to the limits of permissible error for each AMR complex.
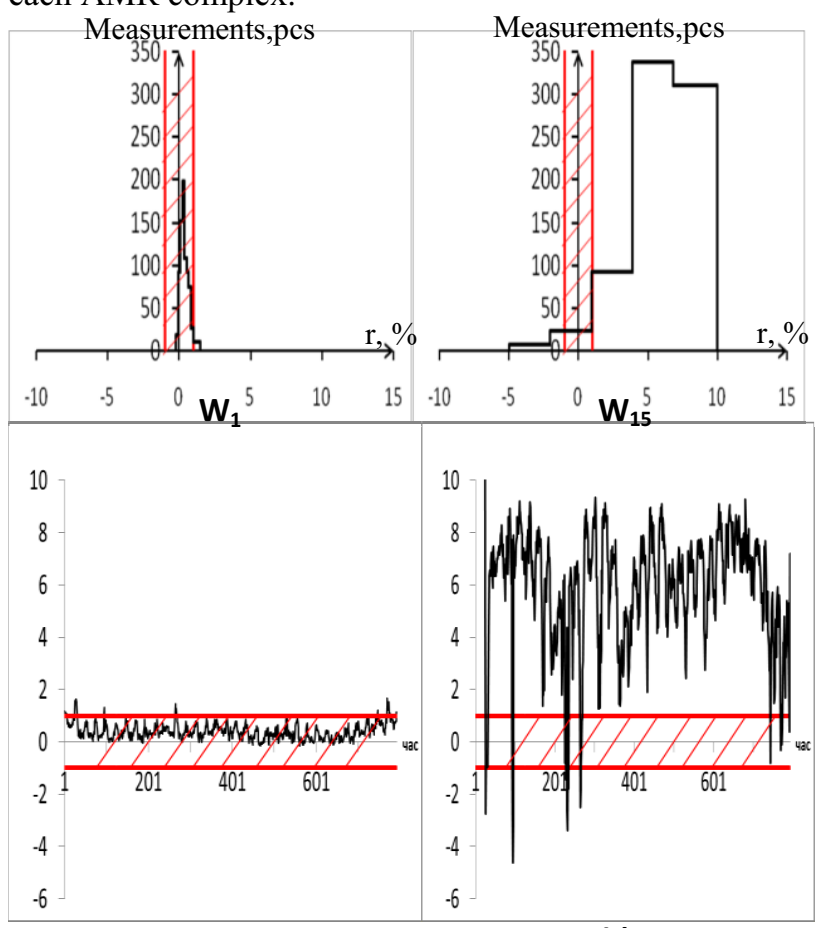

a)

b)

Figure 3. Graphical representation of the calculation results. Evaluation of statistical error components.

Fig. 3a shows the calculation results for AMR complex $W_{l}$. It functions within its accuracy class and do not need recalibration.

Fig. 3 b) presents other AMR complex $W_{15}$. It has bias that exceeds maximum allowable limits defined by limits of maximum permissible error of AMR complex under consideration. Complex number 15 requires the attention of specialists to eliminate this error.

\section{Conclusions}

Application of verifying equations technique allows to evaluate the reliability of demanding electrical energy measurements. It also enables to calculate the estimate of energy flow. The error of this estimate is lower than the error of the actual measurement. Reduction is larger, the higher the information redundancy of AMR system.

Application of the proposed method allows to mathematically evaluate the statistical characteristics of the individual AMR complexes errors that identify the complexes operating out of the permissible errors bounds.

\section{Acknowledgement}

The work was supported by Act 211 Government of the Russian Federation, contract № 02.A03.21.0006 and the Ministry of Education and Science of Russian 
Federation (in the framework of state assignment, №13.1928.2014/K (project №1928)).

\section{References}

1. A. Abur, A.G. Exposito Power System State Estimation. Markel Dekker inc., New York. 2004.

2. A. Monticelli. State Estimation in Electric Power Systems - a Generalized Approach. Norwell, M A: Kluwer, 1990.

3. F.C. Schweppe, J. Douglas, D. Rom. Power System State Estimation, Part I - Exact Model, IEEE Transactions on Power Apparatus and Systems, 89, 1970, pp. 120-125.

4. F.C. Schweppe, J. Douglas, D. Rom. Power System State Estimation, Part II - Approximate Model, IEEE Transactions on Power Apparatus and Systems, 89, 1970, pp. 125-130.

5. A. Monticelli. Electric Power Syaytem State Estimation. Proceedings of the IEEE, 88, No.2, pp. 262-282 (2000).

6. A. Monticelli, F.F. Wu, M. Yen. Multiple Bad Data Identification for State Estimation by Combinatorial Optimization. Proceesings of the PICA Conference, pp. 452-460 (1985).

7. X. Nian-De, W. Shi-Ying, Y. Er-Keng. A new approach for Detection and Identification of Multiple Bad Data in Power System State Estimation. IEEE
Transactions on Power Systems, 101, No. 2, pp.454462 (1982).

8. A.Z. Gamm, I.N. Kolosok. Bad data detection in measurements in electric power system. Nauka, Novosibirsk, Sib. Enterpr. RAS (in Russian). (2000).

9. A.V. Pazderin, S.E. Kokin, A.O. Egorov, E.S. Kochneva. Solution of energy flow problem using state estimation technique, ECON Proceedings (Industrial Electronics Conference) 5414817, pp. 1736-1741. (2009).

10. A.O. Egorov, E.S. Kochneva, A.V. Pazderin. Improving of metering information validity on the basis of the control equation method. The first international conference on sustainable power generation and supply, pp.217-222, (2009).

11. A.V. Pazderin, V.O. Samoylenko. A Mathematical Method of Energy Resources Flows Data Validating Using the State Estimation Theory. Renewable Energy \& Power Quality Journal. 10. pp. 348-352. (2012).

12. A.V. Pazderin, A.O. Egorov, S.A. Eroshenko. The energy meters allocation in electric systems on the basis of observability theory, 9th Conference on Environment and Electrical Engineering, EEEIC 2010 5489950, pp. 167-170 (2010).

13. RD 34.09.101-94. Typical instruction for electricity metering in its generation, transmission and distribution. M. ORGRES, S. (in Russian) (1995). 